\title{
Dehydration and Headache
}

\author{
Karissa N. Arca ${ }^{1}$ Rashmi B. Halker Singh ${ }^{1}$ \\ Accepted: 22 May 2021 / Published online: 15 July 2021 \\ (C) The Author(s), under exclusive licence to Springer Science+Business Media, LLC, part of Springer Nature 2021
}

\begin{abstract}
Purpose of Review We define dehydration and its relationship to pain physiology including both primary and secondary headache disorders.

Recent Findings Intravenous fluids administered for acute migraine attacks in an emergency department setting have not been shown to improve pain outcomes. However, increased intravascular volume before diagnostic lumbar puncture may reduce the frequency of post-lumbar puncture headache from iatrogenic spinal fluid leak. Maintenance of euhydration can help treat orthostatic and "coat-hanger" headache due to autonomic disorders. Similarly, prevention of fluid losses can mitigate secondary headaches provoked by dehydration such as cerebral venous thrombosis or pituitary apoplexy.

Summary Dehydration alone may cause headache, but oftentimes exacerbates underlying medical conditions such as primary headache disorders or other conditions dependent on fluid balance.
\end{abstract}

Keywords Headache $\cdot$ Migraine $\cdot$ Dehydration $\cdot$ Hypohydration $\cdot$ Water $\cdot$ POTS

\section{Introduction}

The International Classification of Headache Disorders 3rd edition (ICHD-3) does not define a specific headache syndrome related to dehydration. However, this phenomenon can be deemed a secondary headache and considered under headache attributed to disorders of homeostasis [1]. Dehydration is the active process of losing body water; when there is a water deficit in the body, it is referred to as hypohydration [2]. Conditions that cause dehydration include but are not limited to medications (diuretics), diarrhea or emesis, sweat, cold, altitude, and lack of adequate water intake [2]. Despite the known relationship between dehydration, hypohydration, and headache, the pathophysiology is still not fully understood. It has been suggested that water deficit in the body may result in dural venous stretching (MonroKelli doctrine) and thereby provoke headache. In this case,

This article is part of the Topical Collection on Uncommon and/or Unusual Headaches and Syndromes

Karissa N. Arca

Arca.karissa@mayo.edu

1 Mayo Clinic Arizona, 13400 E Shea Blvd, Scottsdale, AZ 85259, USA restoration of intravascular volume could also abort headache [3]. The underlying mechanism for dehydration-related headache is probably variable and multi-factorial depending on the individual and other associated health conditions such as primary headache or autonomic disorders. In this review, we examine the current evidence detailing the association between dehydration and headache with attention to both primary and secondary headache disorders. We also describe headache related to orthostatic hypotension, postural orthostatic tachycardia syndrome, and cerebrospinal fluid (CSF) hypotension which can be exacerbated by dehydration. Finally, we review general recommendations for treatment and prevention of dehydration related headache.

\section{Dehydration and Pain}

Exaggerated bodily response to pain in the dehydrated state has been demonstrated in two interventional studies. These investigations evaluated pain response to the cold pressor test (immersion of upper or lower limbs in cold water) in a dehydrated compared to euhydrated state and found that pain response was increased in subjects who were dehydrated [4]. Furthermore, functional magnetic resonance imaging (fMRI) in dehydrated subjects undergoing the cold pressor test 
demonstrated enhanced activation in central nervous system pain networks as well as reduced pain threshold to the cold pressor test compared to the rehydrated state [5]. Notably, these studies were performed in healthy adults and there cannot be a direct extrapolation to the migraine or headache population. However, one may speculate that the threshold for a migraine or other headache attack could similarly be reduced when dehydrated. From a headache perspective, inadequate fluid intake leads to hypertonicity and subsequent "brain dehydration" which can exert traction on the pain-sensitive meninges and vascular structures [6, 7]. The exact mechanism of headache due to dehydration is not fully understood.

\section{Dehydration in Primary Headache Disorders}

Dehydration and hypohydration can be difficult to characterize in clinical trials or patient office visits. Dehydration can be measured by percent of body mass loss, plasma osmolality, urine osmolality, or specific gravity [2]. Most studies investigating dehydration as a source or trigger for headache do not include these measurements. A small randomized pilot study from 2005 studied the effect of an additional intake of $1.5 \mathrm{~L}$ of water on headache-hours per 2 weeks in subjects with migraine or tension-type headache. Compared to controls, the group with increased water intake did have reduced number of headache-hours, headache attacks, and acute medication use; however, quality of life did not appear to be improved and the results were not statistically significant [8]. A followup study included a slightly larger randomized controlled cohort of patients who were allocated to either increasing water intake by $1.5 \mathrm{~L}$ daily or to the control (no increase in water consumption) [9]. Reduction in headache days did not significantly differ between the experimental group and the controls though the intervention arm reported improved quality of life [9]. Understanding the relationship between hydration status and primary headache may require separation of migraine and tension-type headache, or at least a post hoc analysis of how these two groups responded to hydration. To further illuminate this point, a recent cross-sectional study examined the association between water intake and migraine in 256 women with migraine [10]. Women who drank more water and total fluids (around $2 \mathrm{~L}$ per day) had reduced severity, duration, and frequency of migraine attacks as well as reduced attackassociated disability compared to those who drank less [10].

"Water deprivation headache" has been proposed as a primary headache disorder though not currently recognized as such in the ICHD-3 [7]. Based on a small case series, there were no characterizable features of water deprivation headache other than relief within minutes of consuming fluids [7]. Fasting is a potential etiology of water deprivation. Fasting headache tends to occur with $16 \mathrm{~h}$ of initiation of fast and usually resolves $72 \mathrm{~h}$ after breaking the fast [11]. These time criteria used to be included in the international classification of headache disorders; however, the criteria have changed to include headache that starts after $8 \mathrm{~h}$ of fasting and duration of headache is no longer part of the criteria [12]. During Ramadan, fasting from food and beverage (including water) is practiced from dusk until dawn. Among 32 practicing Muslim people with migraine, increased migraine attacks were noted during Ramadan compared to the following month (9.4 \pm 4.3 compared to $3.7 \pm 2.1$ attacks). However, investigators did not document the quantity of fluid intake during and after Ramadan [13]. Asking patients about the practice of fasting whether it is for religious, cultural, or personal reasons may help the clinician further understand the patient's headache attack characteristics and devise an individualized plan for treatment.

While consumption of alcohol is typically thought of as dehydrating, classic alcohol-induced or withdrawal headache as defined by the ICHD-3 do not seem to be directly associated with hydration status [14]. Up to $60 \%$ of people with migraine identify alcohol as a trigger $[15,16]$ and induction of oxidative stress by the diuretic properties of alcohol may play a role in triggering a migraine attack [17]. Specifically, elevated plasma levels of arginine vasopressin increase production of superoxide anions which impair cerebrovascular autoregulation [17]. For many individuals with migraine who identify alcohol as a trigger, even a small amount is sufficient to start an attack, supporting the idea that this is a separate phenomenon from hydration status.

\section{Dehydration in an Acute Migraine Attack and Status Migrainosus}

Dehydration can occur during an acute migraine attack or status migrainosus due to nausea, poor oral intake, and emesis. In a 1994 survey of self-reported people with migraine, emesis occurred in almost $70 \%$ of people with migraine. Nausea alone was even more prominent with greater than $90 \%$ of people experiencing this symptom. Both nausea and emesis were reported to limit the ability to take oral migraine medications [18]. Inability to take medications can lead to a prolonged migraine attack, further reducing oral fluid intake. Analysis of emergency department visits for headache or migraine found that administration of intravenous fluids prolonged emergency department stays [19] and when administered with metoclopramide did not provide additional head pain benefit compared to metoclopramide alone [20]. However, patients should be treated for dehydration if clinically evident by history, exam, or lab studies [21]. An additional recent randomized control trial of 50 patients with migraine in the emergency department evaluated headache response to $1 \mathrm{~L}$ of normal saline over $1 \mathrm{~h}$ compared to saline at $10 \mathrm{~mL} / \mathrm{h}$ over $1 \mathrm{~h}$; both groups received prochlorperazine and diphenhydramine. The therapy was blinded to everyone except the patient's nurse. There was no statistically significant 
change in pain scores at $1 \mathrm{~h}$ or $2 \mathrm{~h}$ in the treatment arm compared to the control group [22•]. This was a particularly important study because it was the first prospective evaluation of pain resolve to intravenous fluids in migraine. While the studies investigating the use of intravenous fluids in the treatment of acute migraine attacks in the emergency department have not yet identified a therapeutic role for intravenous fluids, additional research would benefit from objective documentation of volume status.

\section{Secondary Headache due to Dehydration}

Categorization of headache due to dehydration as the primary cause generally best fits under the ICHD-3 diagnosis "Headache attributed to other disorder of hemostasis" [1]. However, dehydration can also cause or worsen other secondary headache disorders. For instance, active duty military personnel interviewed about high altitude headache while training at a high-altitude site found that this type of headache was significantly associated with dehydration based on a univariate analysis (though the association did not remain significant with multivariate analysis) [23]. Similarly, exercise can lead to hypohydration and subsequent headache. A case series of 8 adult men completing exercise under various levels of hypohydration found that headache was more frequently reported with worse states of hypohydration [24]. In both these studies, however, it is unclear if any of these subjects had baseline history of migraine or other primary headache disorders.

Dehydration is a risk factor for thrombosis, particularly cerebral venous sinus thrombosis (CVST) which can present as a thunderclap-onset headache. A 7-year retrospective crosssectional study was conducted in Iran and found higher prevalence of CVST during the months of Ramadan compared to the other months in the lunar calendar [25]. The authors suspect this is due to elevated blood viscosity from dehydration during the months of Ramadan [25]. High estrogen states such as pregnancy or combined hormonal contraceptive use can also increase the risk of thrombosis $[25,26]$. In a retrospective analysis of 22 women with CVST during pregnancy or the postpartum period, dehydration was a predisposing factor during pregnancy. While dehydration may be a risk factor in the post-partum period as well, it was not identified in this cohort of patients [26]. In children, dehydration alone or in conjunction with infection are risk factors for CVST [27]. Another cause of thunderclap-onset headache, pituitary apoplexy, can occur due to dehydration. One case report documents pituitary apoplexy due to severe hyperglycemia. In this case, elevated serum glucose resulted in hyperosmolarity and dehydration which altered the microvasculature surrounding the pituitary [28]. Particularly in patients who present with dehydration and thunderclap headache, clinicians need to maintain a high index of suspicion for secondary causes.

Historically, dehydration was considered a mechanism for spontaneous CSF hypotension due to reduced blood flow and subsequent reduction CSF secretion [29]. However, evidence to support this theory is limited. More often in clinical care, healthcare providers encounter iatrogenic CSF hypotension and post-dural puncture headache following a lumbar puncture (LP). It has been debated whether supplemental fluid administration reduces the incidence of post-dural puncture headache and a 2016 Cochrane review determined that "the role of fluid supplementation in the prevention of post-dural puncture headache is unclear" [30]. Subsequent to this Cochrane review, a prospective parallel study of patients undergoing diagnostic LP were randomized to a control group and a pre-hydration group instructed to drink $1.5 \mathrm{~L}$ of fluids in the $24 \mathrm{~h}$ preceding LP in addition to $1 \mathrm{~L}$ normal saline intravenous bolus [31•]. Post-LP headache occurred in $37.3 \%$ of patients in the control group compared to $15.4 \%$ in the prehydration group. Post-LP headache also occurred more frequently in younger patients and those with prior history of headache. The authors speculate that elevated ICP (demonstrated by transcranial Doppler pulsatile index) as the result pre-LP hydration may have been protective against developing post-lumbar puncture headache. Like post-LP headache, autonomic disorders can cause orthostatic headache and individuals with these conditions tend to be sensitive to fluid shifts and dehydration.

Considering the ongoing COVID-19 pandemic, it is also worth noting that in 172 individuals with COVID-19 infection-related headache, dehydration was found to be associated with increased frequency and severity of head pain. In this study, dehydration was determined by measurements of serum osmolality [32•].

\section{Autonomic Disorders and Headache}

Dehydration can predispose to orthostatic intolerance and hypotension. A "coat hanger" distribution of headache affecting the occiput, neck, and shoulders in patients with autonomic failure and orthostatic hypotension was described in 1994 [33]. Orthostatic hypotension can occur as the result of an autonomic or cardiac disorder, hypovolemia, or medications [34]. Neurologists and headache specialists can use the orthostatic nature of headache and this classical distribution of pain in a "coat hanger" distribution to delineate a secondary cause of headache, specifically "head and/or neck pain attributed to orthostatic (postural) hypotension" [35]. To make this diagnosis, orthostatic hypotension must be documented, and typically head pain occurs in the upright posture and resolves in a supine position [35]. The pathophysiology underlying the "coat hanger headache" is likely multifactorial. Some have 
attributed the pain to ischemia of the trapezius and other neck muscles. However, the lack of ischemic complications of other vulnerable structures above the neck makes it unlikely that this is the only mechanism of pain [34]. Nociceptors present in the posterior fossa arteries, venous sinuses, and dura as well as the musculoskeletal components of the neck may become activated by a complex cascade of events triggered by orthostatic hypotension [36]. Interestingly, a case series of 34 adults who experienced headache associated with water deprivation did not consistently demonstrate an occipitally predominant headache or consistent provocation by upright posture though mechanistically it was proposed to occur similarly to the "coat hanger" headache [7]. Perhaps the duration of water deprivation was not significant enough to alter volume status and cause orthostatic intolerance or hypotension.

Postural orthostatic tachycardia syndrome (POTS) is another autonomic disorder that is influenced by intravascular volume status; however, unlike autonomic failure, POTS is not associated with orthostatic hypotension [37]. POTS can be thought of as a syndrome that is the cumulative result of various disorders and risk factors, one of which includes migraine [38]. Central hypovolemia (pooling of plasma volume in abdomen and lower limbs) in POTS may be due to a combination of sympathetic over-activity and denervation whereas other people with POTS may have renal and neuroendocrine dysfunction resulting in hypovolemia [39]. Some patients with POTS experience an orthostatic headache, the mechanism of which is also not fully understood. Unlike the postural headache with orthostatic hypotension, POTS-related orthostatic headache is not classically in a "coat hanger" distribution [37]. While most people with POTS have migraine which can have postural sensitivity, the duration of orthostatic headache in POTS is typically shorter than migraine. Additionally, orthostatic headache in POTS can take minutes to develop whereas positional change in migraine usually immediately worsens headache [40]. Recognition in the variability of headache presentation in autonomic disorders can help with accurate diagnosis and treatment.

\section{Treatment for Dehydration-Related Headache}

Treatment of dehydration-related headache is seemingly simple - give fluids. While this can be effective for "water deprivation headache," the best treatment approach for other headache types may be to address the underlying cause of dehydration or the medical comorbidity being aggravated by dehydration. During an acute migraine attack or status migrainosus, dehydration may occur due to poor oral intake or volume loss from emesis. There is no data to support the use of intravenous fluids in emergency department visits for acute migraine; rather, the use of an intravenous anti-emetic may be helpful [20]. On the other hand, long-term management of migraine may benefit from adequate fluid intake ( $\geq 2 \mathrm{~L}$ per day) in addition to other lifestyle measures such as adequate sleep, food intake, stress management, and avoiding triggers such as alcohol $[9,10,13,17]$. Clinicians should be aware that some cultural and religious practices include fasting which may provoke or exacerbate headache attacks, particularly migraine. It may be helpful during the initial intake visit to query if a patient routinely practices fasting for any reason. For episodic periods of fasting in a patient with migraine not currently using a preventive medication, initiation of a once-daily oral preventive medication with rapid or no titration may be beneficial. For acute treatment, long-acting triptans or NSAIDs may be preferred [13].

Prevention of dehydration is the best strategy for most of the secondary headache disorders provoked by loss of body water. Maintaining adequate hydration may help with altitude and exercise-related headache. Furthermore, it may prevent the development of a hypercoagulable or hyperosmolar state. There is also some evidence to suggest that pre-treatment with oral and intravenous fluids may prevent post-lumbar puncture headache $[31 \bullet]$.

Finally, in people with autonomic disorders and prominent orthostatic symptoms such as headache, lifestyle modifications are the cornerstone to treatment. From a fluid perspective, these include increasing water intake to 2-3 L/day and salt $(\mathrm{NaCl})$ intake up to $10 \mathrm{~g}$ daily. Eating small meals throughout the day and utilizing compression stockings or abdominal binders can prevent blood pooling. Graded aerobic exercise is also key to build muscle and prevent deconditioning [41]. When symptoms are severe or remain bothersome despite lifestyle measures, pharmacotherapy should be utilized. In orthostatic hypotension and autonomic failure, fludrocortisone can be helpful if the patient is volume depleted, whereas droxidopa or midodrine are effective for increasing peripheral vascular resistance [42]. Patients with POTS may also benefit from these medications if blood pressure stabilization is needed to improve symptoms; otherwise beta-blockers or ivabradine can be helpful [39].

\section{Conclusion}

Dehydration can cause and exacerbate various headache disorders, the presentations of which are extremely variable. While prevention of dehydration seems to be the best treatment, creative strategies may need to be utilized for lifestyle conditions such as fasting that may provoke dehydrationrelated headache attacks. Further research investigating the relationship between dehydration and headache would benefit from objective documentation of dehydration. In patients who present with dehydration and thunderclap headache, it is important to do a thorough workup for secondary causes. Given that autonomic conditions including POTS can be associated 
with both dehydration and headache, keep these diagnoses in mind when evaluating patients who present with orthostatic symptoms and headache, as a combination of lifestyle modifications and medications can give significant improvement.

\section{Compliance with Ethical Standards}

Conflict of Interest Rashmi Halker Singh declares no conflict of interest.

Karissa Arca reports personal speaker fees from Miles from Migraine, separate from this submission.

Human and Animal Rights and Informed Consent This article does not contain any studies with human or animal subjects performed by any of the authors.

\section{References}

Papers of particular interest, published recently, have been highlighted as:

- Of importance

1. Gobel $\mathrm{H} 10.7$ Headache attributed to other disorder of homoeostasis. In: ICHD-3 The International Classification of Headache Disorders 3rd edition. https://ichd-3.org/10-headacheattributed-to-disorder-of-homoeostasis/10-7-headache-attributedto-other-disorder-of-homoeostasis/. Accessed 27 Feb 2021

2. Liska D, Mah E, Brisbois T, Barrios PL, Baker LB, Spriet LL. Narrative review of hydration and selected health outcomes in the general population. Nutrients. 2019;11:11. https://doi.org/10.3390/ nu1 1010070

3. Bhatia MS, Gupta R, Srivastava S. Migraine associated with water deprivation and progressive myopia. Cephalalgia. 2006;26:758-60. https://doi.org/10.1111/j.1468-2982.2006.01083.x.

4. Ogino Y, Kakeda T, Nakamura K, Saito S. Dehydration enhances pain-evoked activation in the human brain compared with rehydration. Anesth Analg. 2014;118:1317-25. https://doi.org/10.1213/ ANE.0b013e3182a9b028.

5. Perry BG, Bear TLK, Lucas SJE, Mündel T. Mild dehydration modifies the cerebrovascular response to the cold pressor test. Exp Physiol. 2016;101:135-42. https://doi.org/10.1113/EP085449.

6. van der Jagt M. Fluid management of the neurological patient: a concise review. Crit Care. 2016;20:126. https://doi.org/10.1186/ s13054-016-1309-2.

7. Blau JN, Kell CA, Sperling JM. Water-deprivation headache: a new headache with two variants. Headache. 2004;44:79-83. https://doi. org/10.1111/j.1526-4610.2004.04014.x.

8. Spigt MG, Kuijper EC, Schayck CP, Troost J, Knipschild PG, Linssen VM, et al. Increasing the daily water intake for the prophylactic treatment of headache: a pilot trial. Eur J Neurol. 2005;12: 715-8. https://doi.org/10.1111/j.1468-1331.2005.01081.x.

9. Spigt M, Weerkamp N, Troost J, van Schayck CP, Knottnerus JA. A randomized trial on the effects of regular water intake in patients with recurrent headaches. Fam Pract. 2012;29:370-5. https://doi. org/10.1093/fampra/cmr112.

10. Khorsha F, Mirzababaei A, Togha M, Mirzaei K. Association of drinking water and migraine headache severity. J Clin Neurosci. 2020;77:81-4. https://doi.org/10.1016/j.jocn.2020.05.034.
11. Torelli P, Manzoni GC. Fasting headache. Curr Pain Headache Rep. 2010;14:284-91. https://doi.org/10.1007/s11916-010-0119-5.

12. Gobel H 10.5 Headache attributed to fasting. In: ICHD-3 The International Classification of Headache Disorders 3rd edition. https://ichd-3.org/10-headache-attributed-to-disorder-ofhomoeostasis/10-5-headache-attributed-to-fasting/. Accessed 7 Feb 2021

13. Abu-Salameh I, Plakht Y, Ifergane G. Migraine exacerbation during Ramadan fasting. J Headache Pain. 2010;11:513-7. https://doi. org/10.1007/s10194-010-0242-z.

14. Panconesi A, Bartolozzi ML, Guidi L. Alcohol and migraine: what should we tell patients? Curr Pain Headache Rep. 2011;15:177-84. https://doi.org/10.1007/s11916-011-0184-4.

15. Panconesi A. Alcohol and migraine: trigger factor, consumption, mechanisms. A review. J Headache Pain. 2008;9:19-27. https://doi. org/10.1007/s10194-008-0006-1.

16. Turner DP, Houle TT. Influences on headache trigger beliefs and perceptions. Cephalalgia. 2018;38:1545-53. https://doi.org/10. $1177 / 0333102417739310$.

17. Borkum JM. Migraine triggers and oxidative stress: a narrative review and synthesis. Headache. 2016;56:12-35. https://doi.org/ 10.1111/head.12725.

18. Silberstein SD. Migraine symptoms: results of a survey of selfreported migraineurs. Headache. 1995;35:387-96. https://doi.org/ 10.1111/j.1526-4610.1995.hed3507387.x.

19. Jones CW, Gaughan JP, McLean SA. Epidemiology of intravenous fluid use for headache treatment: findings from the National Hospital Ambulatory Medical Care Survey. Am J Emerg Med. 2017;35:778-81. https://doi.org/10.1016/j.ajem.2017.01.030.

20. Balbin JEB, Nerenberg R, Baratloo A, Friedman BW. Intravenous fluids for migraine: a post hoc analysis of clinical trial data. Am J Emerg Med. 2016;34:713-6. https://doi.org/10.1016/j.ajem.2015. 12.080 .

21. Long BJ, Koyfman A. Benign Headache Management in the Emergency Department. J Emerg Med. 2018;54:458-68. https:// doi.org/10.1016/j.jemermed.2017.12.023.

22. Jones CW, Remboski LB, Freeze B, et al. Intravenous fluid for the treatment of emergency department patients with migraine headache: a randomized controlled trial. Ann Emerg Med. 2019;73: 150-6. https://doi.org/10.1016/j.annemergmed.2018.09.004 First prospective evaluation of pain response to intravenous fluids in migraine.

23. Norris JN, Viirre E, Aralis H, Sracic MK, Thomas D, Gertsch JH. High altitude headache and acute mountain sickness at moderate elevations in a military population during battalion-level training exercises. Mil Med. 2012;177:917-23. https://doi.org/10.7205/ milmed-d-12-00007.

24. Richardson A, Watt P, Maxwell N. The effect of hypohydration severity on the physiological, psychological and renal hormonal responses to hypoxic exercise. Eur J Appl Physiol. 2009;106: 123-30. https://doi.org/10.1007/s00421-009-0997-6.

25. Javanmardi H, Safari A, Borhani-Haghighi A. Effect of Ramadan fasting in incidence of cerebral venous sinus thrombosis. Int $\mathrm{J}$ Stroke. 2018;13:NP2. https://doi.org/10.1177/1747493017743799.

26. Gao H, Yang B-J, Jin L-P, Jia X-F. Predisposing factors, diagnosis, treatment and prognosis of cerebral venous thrombosis during pregnancy and postpartum: a case-control study. Chin Med J. 2011;124: 4198-204.

27. Sébire G, Tabarki B, Saunders DE, et al. Cerebral venous sinus thrombosis in children: risk factors, presentation, diagnosis and outcome. Brain. 2005;128:477-89. https://doi.org/10.1093/brain/ awh412.

28. Mittal A, Mishra S, Yadav K, Rajput R. Uncontrolled diabetes as a rare presenting cause of pituitary apoplexy. BMJ Case Rep. 2019;12:12. https://doi.org/10.1136/bcr-2018-228161. 
29. http://fyra.io Spontaneous Intracranial Hypotension. In: Practical Neurology. https://practicalneurology.com/articles/2014-may/ spontaneous-intracranial-hypotension. Accessed 22 Feb 2021

30. Arevalo-Rodriguez I, Ciapponi A, Roqué i Figuls M, et al. Posture and fluids for preventing post-dural puncture headache. Cochrane Database Syst Rev. 2016;3:CD009199. https://doi.org/10.1002/ 14651858.CD009199.pub3.

31. Nowaczewska M, Kukulska-Pawluczuk B, Kaźmierczak H, Pawlak-Osińska K. Post-lumbar puncture headache - does hydration before puncture prevent headache and affect cerebral blood flow? J Clin Med. 2019;8:1710. https://doi.org/10.3390/ jcm8101710 High quality study investigating the relationship to hydration and post-LP headache following a 2016 Cochrane review.

32. Magdy R, Hussein M, Ragaie C, et al. Characteristics of headache attributed to COVID-19 infection and predictors of its frequency and intensity: a cross sectional study. Cephalalgia. 2020;40:142231. https://doi.org/10.1177/0333102420965140 Pertinent to the ongoing pandemic, discusses relationship of COVID-19 headache and dehydration.

33. Robertson D, Kincaid DW, Haile V, Robertson RM. The head and neck discomfort of autonomic failure: an unrecognized aetiology of headache. Clin Auton Res. 1994;4:99-103. https://doi.org/10.1007/ BF01845772.

34. Khurana RK. Syncope and Headache. Curr Pain Headache Rep. 2018;22:54. https://doi.org/10.1007/s11916-018-0706-4.

35. Gobel H A10.7 Head and/or neck pain attributed to orthostatic (postural) hypotension. In: ICHD-3 The International Classification of Headache Disorders 3rd edition. https://ichd-3. org/appendix/a10-headache-attributed-to-disorder-ofhomoeostasis/a10-7-head-andor-neck-pain-attributed-toorthostatic-postural-hypotension/. Accessed 27 Feb 2021

36. Khurana RK. Coat-hanger ache in orthostatic hypotension. Cephalalgia. 2012;32:731-7. https://doi.org/10.1177/ 0333102412449932.

37. Mokri B, Low PA. Orthostatic headaches without CSF leak in postural tachycardia syndrome. Neurology. 2003;61:980-2. https://doi.org/10.1212/01.wnl.0000085868.37963.7d.

38. Arnold AC, Ng J, Raj SR. Postural tachycardia syndrome - diagnosis, physiology, and prognosis. Auton Neurosci. 2018;215:3-11. https://doi.org/10.1016/j.autneu.2018.02.005.

39. Fedorowski A. Postural orthostatic tachycardia syndrome: clinical presentation, aetiology and management. J Intern Med. 2019;285: 352-66. https://doi.org/10.1111/joim.12852.

40. Khurana RK, Eisenberg L. Orthostatic and non-orthostatic headache in postural tachycardia syndrome. Cephalalgia. 2011;31: 409-15. https://doi.org/10.1177/0333102410382792.

41. Wig R, Oakley CB. Dysautonomia and headache in the pediatric population. Headache. 2019;59:1582-8. https://doi.org/10.1111/ head.13659.

42. Palma J-A, Kaufmann H. Treatment of autonomic dysfunction in Parkinson disease and other synucleinopathies. Mov Disord. 2018;33:372-90. https://doi.org/10.1002/mds.27344.

Publisher's Note Springer Nature remains neutral with regard to jurisdictional claims in published maps and institutional affiliations. 